\title{
miR-223 reverses the resistance of EGFR-TKIs through IGF1R/PI3K/Akt signaling pathway
}

\author{
JING HAN $^{1}$, FENGYI ZHAO ${ }^{1}$, JING ZHANG $^{2}$, HAIZHEN ZHU ${ }^{3}$, HU MA $^{4}$, \\ XUETAO LI ${ }^{1}$, LINA PENG ${ }^{1}$, JIANGUO SUN ${ }^{1}$ and ZHENGTANG CHEN ${ }^{1}$ \\ ${ }^{1}$ Cancer Institute of PLA, Xinqiao Hospital, Third Military Medical University, Chongqing 400037; \\ ${ }^{2}$ Department of Oncology, 401 Hospital of the People's Liberation Army, Qingdao, Shandong 266071; \\ ${ }^{3}$ Department of Oncology, Guizhou Provincial People's Hospital, Guiyang, Guizhou 550002; \\ ${ }^{4}$ Department of Oncology, Affiliated Hospital of Zunyi Medical College, Zunyi, Guizhou 563000, P.R. China
}

Received December 22, 2015; Accepted January 30, 2016

DOI: $10.3892 /$ ijo.2016.3401

\begin{abstract}
Acquired resistance to epidermal growth factor receptor (EGFR) tyrosine kinase inhibitors (TKIs), such as gefitinib and erlotinib, is a critical issue for the treatment of EGFR mutant-positive non-small cell lung cancer (NSCLC). Recent evidence supports the role of microRNA-223 (miR-223) in modulating chemotherapeutic drug sensitivity, but its role in the resistance to EGFR-TKIs in NSCLC remains unclear. To this end, we investigated the involvement of miR-223 in erlotinib resistance, using two pairs of TKI-sensitive or resistant cell lines, PC9 vs PC9/ER, and HCC827 vs HCC827/ER, as well as $\mathrm{PC} 9 / \mathrm{CD}_{133^{+}}$, which are lung cancer stem-like cells derived from PC9 cells. Downregulation of miR-223 expression in PC9/ER and PC9/CD133+ cells was detected, and the reverse correlation of miR-233 and insulin-like growth factor 1 receptor (IGF1R) in these cells was also revealed. Next, levels of IGF1R mRNA and p-Akt were significantly reduced in miR-223 stably transfected PC9/ER and PC9/CD133+ cells. However, the sensitivity of PC9/ER and PC9/CD133+ cells to erlotinib was partially restored, after overexpression of miR-223 in those cells. Similar results were also observed in vivo. Furthermore, miR-223-mediated inhibition of the IGF1R/PI3K/Akt signaling pathway may have been reversed by the agonist of IGF1R in miR-223 transfected cells. Our findings indicated that downregulation of miR-223, which can induce activation of the IGF1R/phosphatidylinositol 3-kinase (PI3K)/Akt pathway in PC9/ER and PC9/CD133+ cells, may be responsible for the resistance of PC9/ER and PC9/CD133 ${ }^{+}$
\end{abstract}

Correspondence to: Dr Jianguo Sun and Dr Zhengtang Chen, Cancer Institute of PLA, Xinqiao Hospital, Third Military Medical University, Xinqiao Street No. 2, Chongqing 400037, P.R. China

E-mail: sunjg09@aliyun.com

E-mail:czt05@163.com

Key words: EGFR-TKIo, miR-223, erlotinib, non-small cell lung cancer, lung cancer stem-like cells cells to erlotinib, suggesting that miR-223 is a potential therapeutic target for overcoming EGFR-TKIs resistance.

\section{Introduction}

Non-small cell lung cancer (NSCLC) is the most common type of lung cancer, which accounts for $>80 \%$ of all lung cancer cases. The 5-year overall survival rate remains $<15 \%$ in patients with NSCLC, despite advances in integrative therapies, including surgery, chemotherapy and radiotherapy (1). The administration in a clinical setting of epidermal growth factor receptor (EGFR) tyrosine kinase inhibitors (TKIs), such as gefitinib and erlotinib, have significantly improved the overall survival of cancer patients that harbor somatic mutations in the EGFR gene, such as an in-frame deletion mutation in exon 19 (2). Nevertheless, most tumors develop acquired resistance to EGFR-TKIs after a period of time (a median of 6-12 months) (3) in which the tumors exhibit a dramatic response to gefitinib and erlotinib. Acquired resistance to EGFR-TKIs is usually inevitable, leading to a limitation in treatment efficacy. However, the mechanisms underlying acquired resistance to EGFR-TKIs have not been fully understood.

Since acquired drug resistance is a major problem in cancer treatment, different mechanisms have been proposed for developing novel treatment strategies, one example being the progress in the targeting of lung cancer stem cells (CSCs) in recent years. Eramo et al reported (4) isolation and identification of a CSC population that showed extensive drug resistance from tumor specimens of patients with lung cancer. Another study found that the stem cell factor (SCF) and its receptor c-kit (CD117) were expressed to relative degrees in CSCs. The signal transduction pathways of phosphatidylinositol 3-kinase (PI3K) are involved in SCF/c-kit (CD117) activation. Therefore, the proliferation of CSCs can be inhibited by receptor TKIs (5). However, the markers of CSCs are still controversial. A large number of studies have shown that the cell population of $\mathrm{CD}_{133}{ }^{+}$has more characteristics of CSCs than that of CD133 $(4,6)$. CD133 is currently recognized as a well-known marker for CSCs. This marker has been widely used in the isolation and purification of CSCs. 
Furthermore, evidence has recently shown that microRNAs (miRNAs) also regulate certain genes associated with resistance to chemotherapy and EGFR-TKIs (7-9). Among miRNAs related to drug resistance, miRNA-223 (miR-223) was reported to regulate multiple cellular functions via PI3K/Akt signaling pathways in most literature. Our previous studies also showed that miR-223 expression is reduced in a Lewis lung carcinoma cell line and that insulin-like growth factor 1 receptor (IGF1R) served as a target gene of miR-223. The expression of IGF1R and the activity of Akt, its downstream target, were decreased, while miR-223 was overexpressed, indicating that miR-223 inhibited the invasion and metastasis of Lewis lung carcinoma cells by targeting IGF1R-Akt pathway (10). Because of the Akt activity regulated by $\mathrm{P} 13 \mathrm{~K}$, the aberrant activation of IGF1R/P13K/Akt signaling pathway may be the mechanism underlying resistance to EGFR-TKIs. Although several studies showed that IGF1R is implicated in the resistance to chemotherapy, including the targeted therapies, such as EGFR-TKIs $(11,12)$, the correlation between miR-223 and the IGF1R/P13K/Akt pathway in the resistance of EGFR-TKIs has yet to be determined.

In this study, we developed an EGFR-TKI-resistant PC9/ER cell line, in which the percentage of $\mathrm{CD} 133^{+}$cells was so high that isolation of stem cells from CD133+ $\left(\mathrm{PC} 9 / \mathrm{CD} 133^{+}\right.$ cells) was performed. Our study revealed that $\mathrm{CD} 133^{+}$was resistant to erlotinib. The expression of miR-223 in ER and $\mathrm{CD}_{133}{ }^{+}$cells was downregulated, compared to their parent cells. IGF1R was also verified as a target gene of miR-223 in our study. According to these findings, we hypothesized that downregulation of miR-223 expression may induce the activation of the IGF1R/PI3K/Akt signaling pathway, leading to erlotinib resistance. Here, we provide evidence to verify our hypothesis.

\section{Materials and methods}

Cells and reagents. The human lung cancer HCC827 cell line was purchased from ATCC (ATCC ${ }^{\circledR}$ CRL-2868 ${ }^{\mathrm{TM}}$ ). The PC9 cell line, which was derived from a human adenocarcinoma of lung tissue, was preserved in our laboratory. The lung cancer cells were cultured in RPMI-1640 medium containing $10 \%$ fetal bovine serum (Gibco BRL, Carlsbad, CA, USA) and $100 \mathrm{U} / \mathrm{ml}$ penicillin/streptomycin at $37^{\circ} \mathrm{C}$ in a humidified incubator containing $5 \% \mathrm{CO}_{2}$. Erlotinib (OSI-744) was purchased from Selleck Chemicals (Houston, TX, USA). Insulin-like growth factor 1 human recombinant was obtained from ProSpec (ProSpec, Rehovot, Israel). Two erlotinib-resistant lines, namely HCC827/ER and PC9/ER, were developed by applying high-dose $(1-5 \mu \mathrm{M})$ pulses of erlotinib combined with continuous low-dose $(0.01 \mu \mathrm{M})$ administration for $>8$ months (13). To avoid the effects of the drugs, resistant cell lines were cultured in a drug-free medium for $\geq 2$ weeks prior to further experiments.

Isolation of $\mathrm{CD} 133^{+}$cells from the PC9 cell line with paclitaxel treatment. Approximately $10^{6} / \mathrm{ml}$ PC9 cells were suspended in F12 serum-free medium (Hyclone, USA) supplement with $0.4 \%$ bovine serum albumin (BSA; Sigma-Aldrich, St. Louis, MO, USA), insulin $5 \mathrm{~g} / \mathrm{ml}$ (Sigma-Aldrich), human recombinant epidermal growth factor, $20 \mathrm{ng} / \mathrm{ml}$ (PeproTech,
Rehovot, Israel) and basic fibroblast growth factor, $10 \mathrm{ng} / \mathrm{ml}$ (PeproTech). When spheroids emerged, cells were treated for $48 \mathrm{~h}$ with paclitaxel injection (Powerdone, China) at a concentration of $100 \mathrm{nmol} / \mathrm{l}$. The culture medium was replaced with fresh complete medium twice per week until new spheroids emerged. To isolate $\mathrm{CD}_{133^{+}}$cells, spheroids were dissociated into single cells, washed in phosphate-buffered saline (PBS) 3 times and incubated with PE-conjugated monoclonal antibody against human CD133/1 (Miltenyi Biotec), according to the manufacturer's instructions. After incubation for $30 \mathrm{~min}$ at $4^{\circ} \mathrm{C}$, cells were washed in PBS twice and CD133+ cells were sorted by flow cytometry (BD Biosciences).

Construction of stable cell lines with overexpressed miR-223. To stably upregulate miR-223 expression in PC9/ER cells or $\mathrm{PC} 9 / \mathrm{CD} 133^{+}$, lentivirus carrying the miR-223 or negative control vector (empty viral vector; EV) was packaged using the lentiviral packaging kit (Shanghai Genechem Co., Ltd., Shanghai, China), according to the manufacturer's instructions. The green fluorescent protein (GFP) gene was inserted into the packaging system, which allows co-expression of GFP with miR-223. To establish stable cell lines, PC9/ER and $\mathrm{PC} 9 / \mathrm{CD} 133^{+}$cells were infected with lentivirus carrying miR-223 or EV in the presence of polybrene (Sigma-Aldrich).

Luciferase reporter assay and related plasmids. The plasmids of firefly luciferase reporter, IGF1R-WT (wild-type of miR-223 binding site in 3'-UTR of IGF1R) and IGF1R-MU (mutated miR-223 binding site in 3'-UTR of IGF1R) were constructed by Genechem (Shanghai Genechem Co., Ltd., Shanghai, China). The miR-223 mimic and the mimic negative control were obtained from Ribobio (Guangzhou RiboBio Co., Ltd., Guangzhou, China). HEK293 cells were co-transfected with firefly luciferase reporter $(0.05 \mu \mathrm{g})$ and IGF1R plasmid $(0.05 \mu \mathrm{g})$, as well as $0.01 \mu \mathrm{g}$ Renilla luciferase control vector using calcium phosphate transfection. Luciferase activity was measured $36 \mathrm{~h}$ after transfection. To obtain data, Renilla luciferase activity was normalized to firefly luciferase expression, according to the manufacturer's instructions (Dual-Luciferase Reporter Assay System; Promega Corp., Madison, WI, USA).

Cell proliferation assay. Cells that reached mid-log phase growth were spilt and plated into 96-well plates at different densities: 5,000 cells/well (in triplicate) for HCC827 and HCC827/ER cells, but 3,000 cells/well (in triplicate) for PC9, PC9/ER, PC9/ER-miR-223, PC9/ER-EV, PC9/CD133+, $\mathrm{PC} 9 / \mathrm{CD} 133^{+}-\mathrm{miR}-223$, and $\mathrm{PC} 9 / \mathrm{CD} 133^{+}-\mathrm{EV}$ cells. Then cells were cultured for $48 \mathrm{~h}$ in complete medium containing increasing concentrations of $0,0.001,0.01,0.1,1,5$ or $10 \mu \mathrm{M}$ erlotinib. The number of viable cells was quantified using a Cell Counting Kit-8 (CCK-8) (Dojindo Laboratories, Kumamoto, Japan). A total of $10 \mu \mathrm{l}$ CCK- 8 solution was added to each well at the end of treatment, followed by another 2-h incubation. Optical density values at $450 \mathrm{~nm}$ were measured using a microplate reader. Each experiment was independently performed 3 times.

Western blot analysis. Cells were harvested from the experiments by transfection and treatment with inhibitor, washed twice with cold PBS and lysed in RIPA lysis buffer containing 
protease inhibitors. The protein concentration of the lysate was measured. Lysate with a loading buffer was denatured, and then separated by SDS-PAGE, and then electrotransferred to a polyvinylidene difluoride membrane. The membranes were blocked at room temperature for $1 \mathrm{~h}$ in Tris-buffered saline and Tween-20 (TBST) containing 5\% (w/v) BSA (Wuhan Boster Bio-Engineering Co., Ltd., Wuhan, China), before being incubated with the following antibodies: EGFR, p-EGFR, IGF1R, p-IGF1R (1:500, Cell Signaling Technology, Beverly, MA, USA), P70S6K (P70S6 kinase), 70S6K, Akt, p-Akt (Ser473) (1:1,000, Signalway Antibody, Boston, MA, USA), and PTEN (1:500, Wuhan Boster Bio-Engineering Co., Ltd.). After being washed, the membranes were incubated with secondary antibodies conjugated to horseradish peroxidase (Wuhan Boster Bio-Engineering Co., Ltd.). Protein bands were visualized using an enhanced chemiluminescence detection kit (Pierce Biotechnology, Inc., Rockford, IL, USA). $\beta$-actin (Wuhan Boster Bio-Engineering Co., Ltd.) was used as an internal control.

Real-time RT-PCR. Total RNA was extracted from the tissues and harvested cells using RNAiso Reagent Plus (Takara Biotechnology, Dalian, China). Reverse transcription reactions were performed using an RT kit from Takara, according to the manufacturer's protocol. The relative expression level of miR-223 was normalized to that of U6 expression, but the expression levels of other protein coding genes was normalized to that of $\beta$-actin. The primer sets used in quantitative real-time polymerase chain reaction (qRT-PCR) for hsa-miR-223-3p Primer Set and U6 small nuclear ribonucleic acid (snRNA) were purchased from Guangzhou RiboBio Bio-Technique Co., Ltd. The sequences of other primers used in this experiment were as follows: IGF1R: forward, 5'-GGACA GGTCAGAGGGTTTC-3; and reverse, 5'-CTCGTAACTCTT CTCTGTGCC-3'. $\beta$-actin: forward, 5'-GAGCTACGAGCTG CCTGACG-3'; and reverse, 5'-CCTAGAAGCATTTGCGG TGG-3' (10); PTEN: forward, 5'-ACCCCTTCATTGACCTCA ACTA-3'; and reverse, 5'-TCTCGCTCCTGGAAGATGG TGA-3'; C-Met: forward, 5'-TCATTGGTTCCAATCA CAGCTCA-3', reverse, 5'-GCCACCGAGACAGAGGCT AATC-3'. Real-time RT-PCR was performed using the ABI7500 Sequence Detection System. All reactions were performed in triplicate, and all experiments were conducted 3 times independently.

Xenografts. Male BALB/c nude mice (SPF, 6-8-week-old), obtained from the Animal Research Center of Xinqiao Hospital of The Third Military Medical University in China (Chongqing, China), were housed in groups of 5 per cage in a temperature $\left(30 \pm 3^{\circ} \mathrm{C}\right)$ and humidity $(55 \pm 5 \%)$ controlled room. All mice were given water and chow ad libitum at all times. Our animal study was approved by the Institutional Animal Care and Use Committee of Xinqiao Hospital of The Third Military Medical University. The experimental mice were randomized into four groups (5 mice/group). For the tumorigenesis assay, mice were subcutaneously injected with a total of $5 \times 10^{6}$ cells of PC9, or PC9/ER, or PC9/ER transfected with control lentivirus (PC9/ER-EV) or PC9/ER transfected with the miR-223-overexpressing lentivirus (PC9/ER-miR-223), in the right front leg. The tumor volume (V) was calculated according to the formula $\mathrm{V}=\left(\right.$ length $\mathrm{x}$ width $\left.{ }^{2}\right) / 2$. When the tumors reached a mean volume of $\sim 100 \mathrm{~mm}^{3}$ (14), the mice $(\mathrm{n}=5)$ began to receive the erlotinib treatment $(30 \mathrm{mg} / \mathrm{kg} / \mathrm{d}$, via gavage for two weeks) (15). To test the tumor formation ability of $\mathrm{PC} 9 / \mathrm{CD} 133^{+}$cells, nude mice were randomized into three groups ( 3 mice per group). A total of $5 \times 10^{4} \mathrm{PC} 9 / \mathrm{CD} 133^{+}$ or PC9 cells as well as $5 \times 10^{6}$ PC9 cells were subcutaneously injected into nude mice (6-8-week-old) under the front left legs for the tumorigenesis assay. Nude mice were euthanized at the experimental end-point ( 3 weeks after inoculation of tumor cells).

Statistical analysis. Data analysis was performed using SPSS 17.0 software (SPSS, Inc., Chicago, IL, USA). All assays were repeated three times, and the results are expressed as the means \pm SD. The statistical significance of the results between each group was determined using one-way ANOVA. $\mathrm{P}<0.05$ was considered to be significant.

\section{Results}

Establishment of erlotinib-resistant PC9 and HCC827 cells. Two cell lines resistant to EGFR-TKIs, namely PC9/ER and HCC827/ER, were derived from their parental cells through the treatment with high-dose $(1-5 \mu \mathrm{M})$ pulses of erlotinib, combined with continuous low-dose $(0.01 \mu \mathrm{M})$ for $>8$ months. Resistance in the treated cell lines was determined via CCK8 assay, according to the effects of increasing concentrations of erlotinib on cell viability. The half maximal inhibitory concentration $\left(\mathrm{IC}_{50}\right)$ values for erlotinib were 37.4 times higher in PC9/ER cells and 155.4-fold higher in HCC827/ER cells, than in the corresponding parental cells (Fig. 1A). To check the characteristics of those ER resistant cells, several tests were performed, in which the secondary T790M EGFR mutation was not detected in PC9/ER or HCC827/ER cells in the amplification refractory mutation system (ARMS) assay (Fig. 1B). Amplification of the c-Met gene was not found in either PC9/ER or HCC827/ER cells, based on the results of real-time RT-PCR (Fig. 1C). PTEN mRNA expression was decreased by $50 \%$ in HCC $827 / \mathrm{ER}$ cells, compared to its parental HCC827 cells. The expression level of PTEN protein was also downregulated in HCC827/ER cells (Fig. 1E). The downregulation of PTEN may contribute to secondary resistance in HCC827/ER cells. However, no significant differences in PTEN expression were observed between PC9 and PC9/ER cells (Fig. 1D).

Activation of IGF1R/PI3K/Akt pathway in PC9/ER cells. Our study revealed that the acquired resistance of erlotinib in PC9/ER cells was not associated with any known mechanisms related to erlotinib resistance, such as the T790M EGFR mutation, the amplification of the proto-oncogene, receptor tyrosine kinase MET oncogene, or the downregulation of PTEN. As some reports showed that the activation of IGF1R is linked to acquired resistance to EGFR-TKIs (16), we investigated the role of IGF1R in the development of acquired erlotinib resistance in PC9/ER cells. Our results showed that when PC9 and PC9/ER cells were treated with erlotinib at doses of $0,0.001$, and $0.01 \mu \mathrm{M}$ for $72 \mathrm{~h}(17)$, the phosphorylation of EGFR in the parent cells was inhibited by erlotinib, but it was not affected 
A
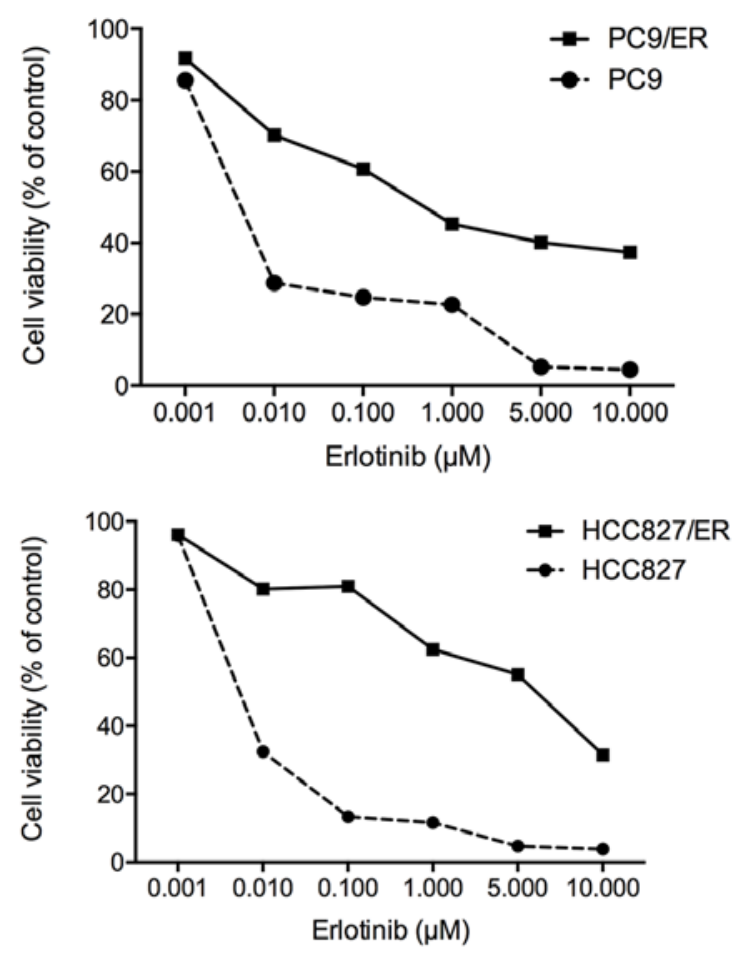

B
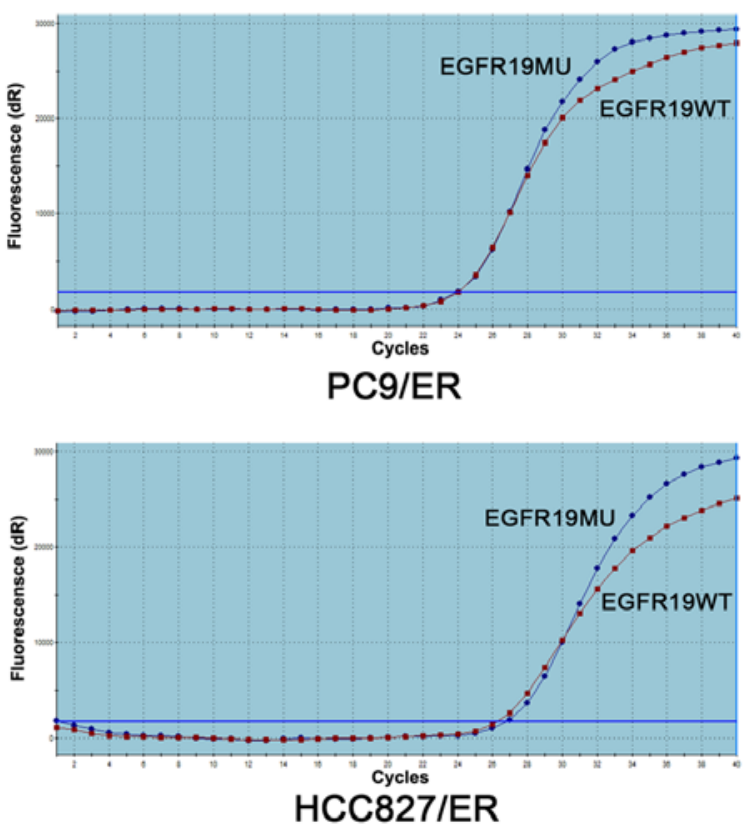

C
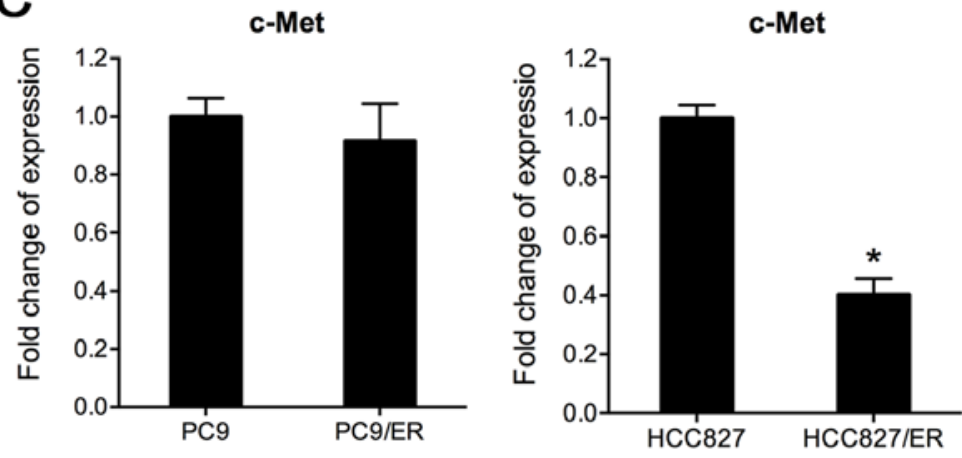

D

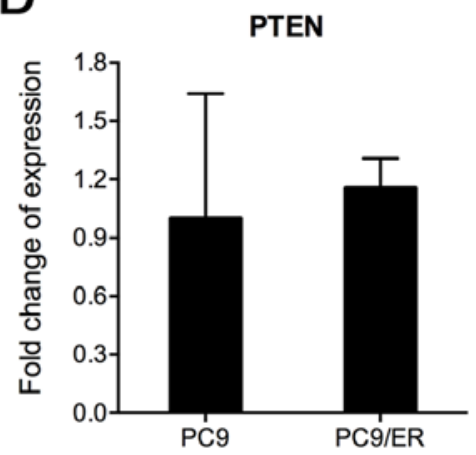

PTEN

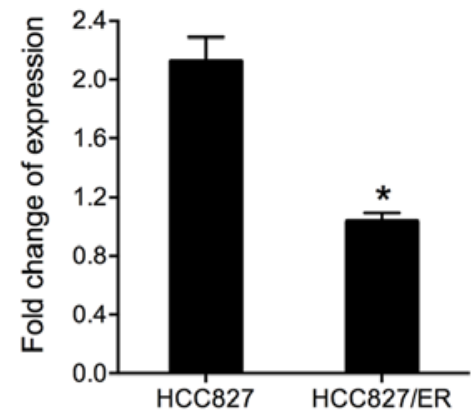

$E$
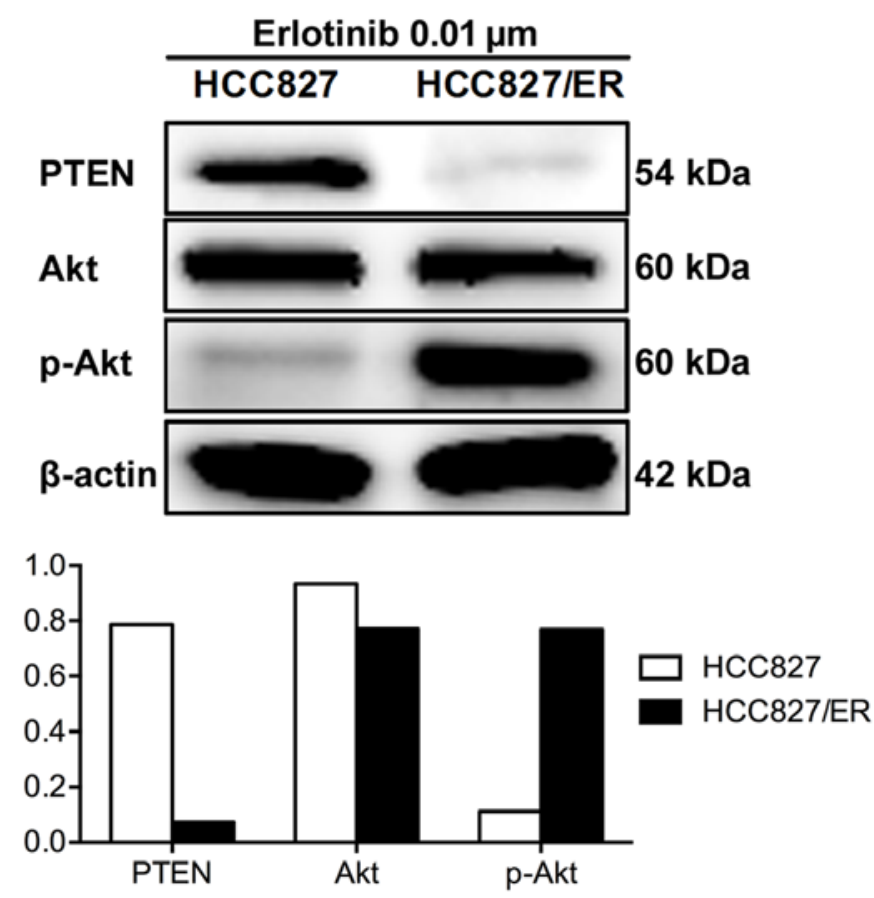

Figure 1. The establishment of erlotinib-resistant PC9 and HCC827 cell lines. (A) Cell viability and IC 50 values of EGFR-TKIs in parental PC9 and HCC827 cell lines as well as in resistant cell lines PC9/ER and HCC827/ER. Cells were treated with indicated concentrations of erlotinib for 72 h in a medium containing $1 \%$ FBS. Cell viability and $\mathrm{IC}_{50}$ values were determined using CCK8 assay. (B) EGFR mutation was detected in PC9/ER and HCC827/ER cell lines using ARMS assay. (C) c-Met gene amplification was detected in PC9/ER and HCC827/ER cell lines via real-time RT-PCR. (D) Expression level of PTEN in PC9/ER and HCC827/ER cells was measured by real-time RT-PCR. (E) Levels of PTEN/Akt signaling molecules in HCC827 and HCC827/ER cells were evaluated by western blot analysis.

in PC9/ER cells (Fig. 2A). Moreover, downstream molecules of the IGF1R/Akt signaling pathway were persistently activated in PC9/ER cells. Therefore, the activation of IGF1R/ $\mathrm{PI} 3 \mathrm{~K} / \mathrm{Akt}$ signaling cascades was involved in the acquired erlotinib resistance of PC9/ER cells. According to this observation, we considered IGF1R as a direct target of miR-223. To verify this speculation, HEK293 cells were transfected with miR-223, mimic control, the wild form 3'-UTR of IGF1R gene 

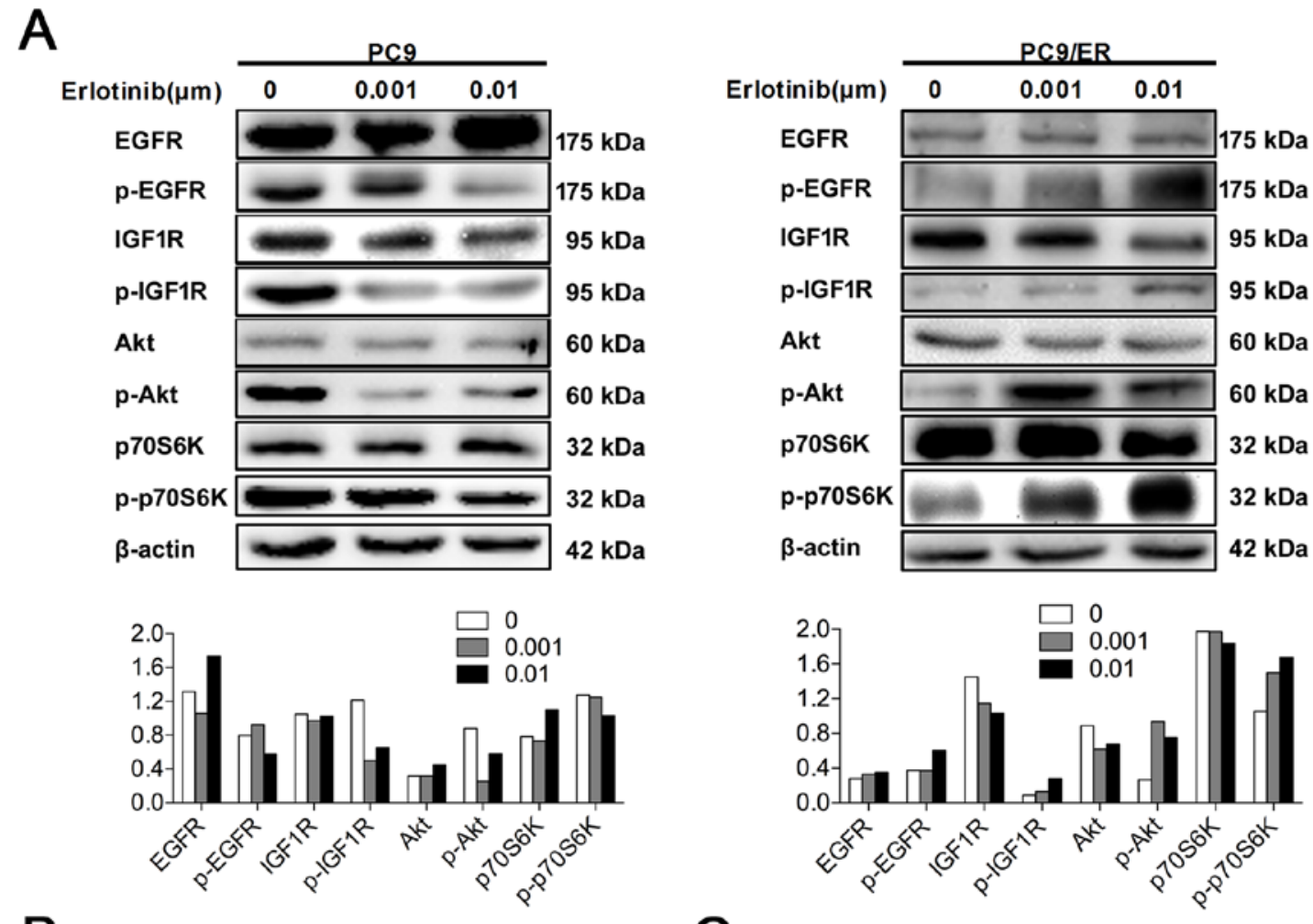

B
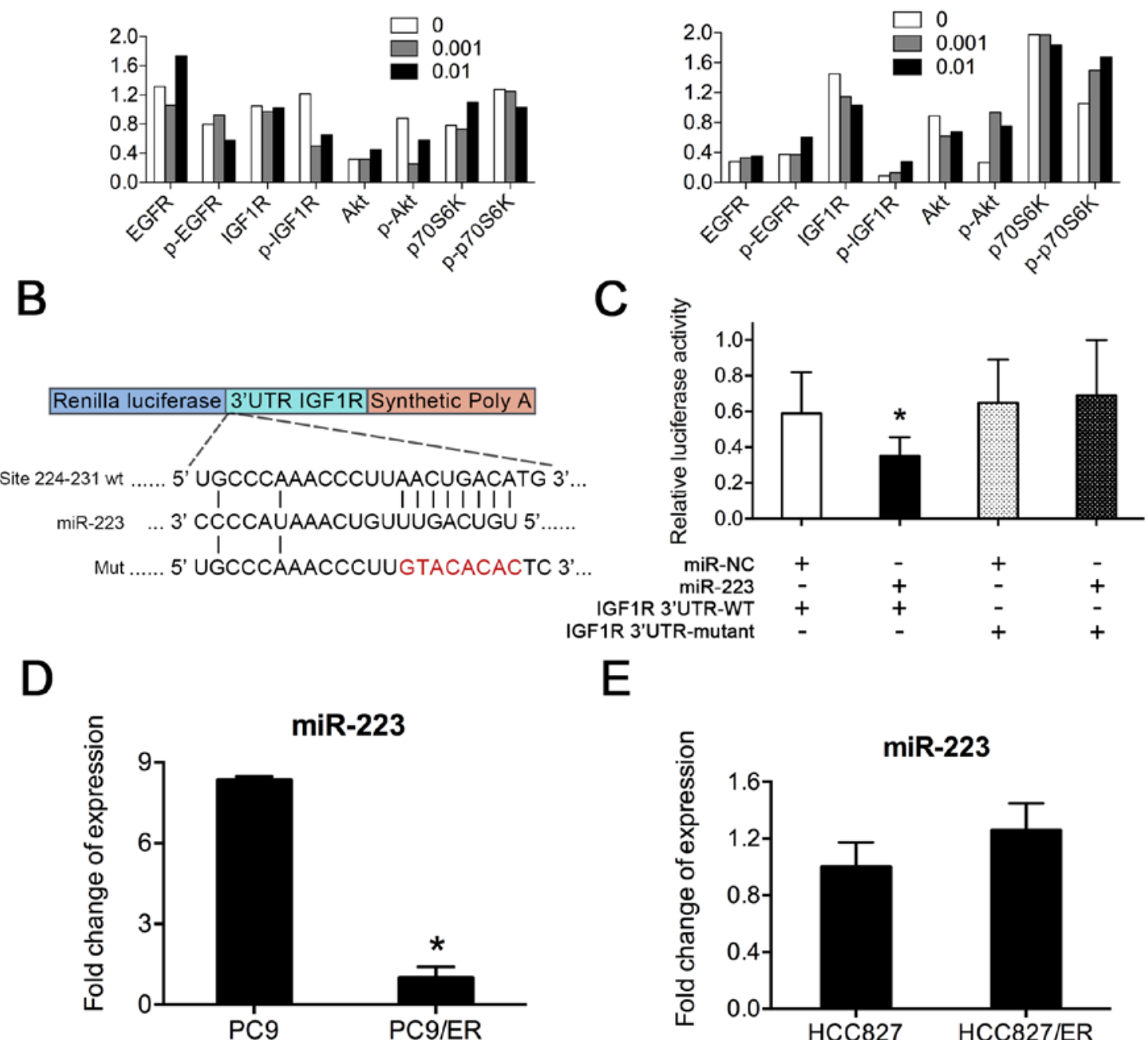

$\mathrm{E}$

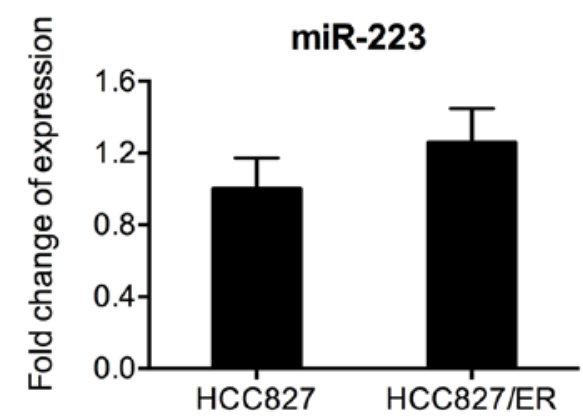

Figure 2. The expression of EGFR-related signaling molecules in PC9 and resistant cell lines. (A) Basal levels of EGFR and EGFR-related signaling molecules in PC9 and PC9/ER cells were evaluated by western blot analysis (28 $\mu \mathrm{g}$ protein of PC9 and $15 \mu \mathrm{g}$ PC9ER were loaded). Effects of erlotinib on EGFR-related signaling molecules were also examined. PC9 and PC9/ER cells were treated with 0.001 or $0.01 \mu \mathrm{M}$ erlotinib for $72 \mathrm{~h}$ in medium containing $1 \%$ FBS (B) Wild-type and nucleotides mutated within IGF-1R mRNA 3'-UTR were cloned into the reporter based on the predicted binding site in the 3'-UTR of IGF-1R with miR-223 seed sequence. (C) Luciferase 3'-UTR reporter assays of miR-223-induced gene silencing effects. (D and E) miR-223 expression in PC9, PC9/ER (D), HCC827and HCC827/ER cells (E). Comparison of miR-223 expression between PC9/ER and PC9 cells, based on data of real-time RT-PCR. No difference in miR-223 expression was observed between HCC827 and HCC827/ER cells.

(3'-UTR-WT), and its mutant form (3'-UTR-MU). The results of luciferase reporter assay showed that the luciferase activity in the group co-transfected with miR-223 and IGF1R-3'-
UTR-WT was significantly lower than that in the group with miR-NC and IGF1R3'-UTR-MU $(\mathrm{P}<0.05)$, implying the inhibitory effect of miR-223 on 3'-UTR of the IGF1R gene, which 

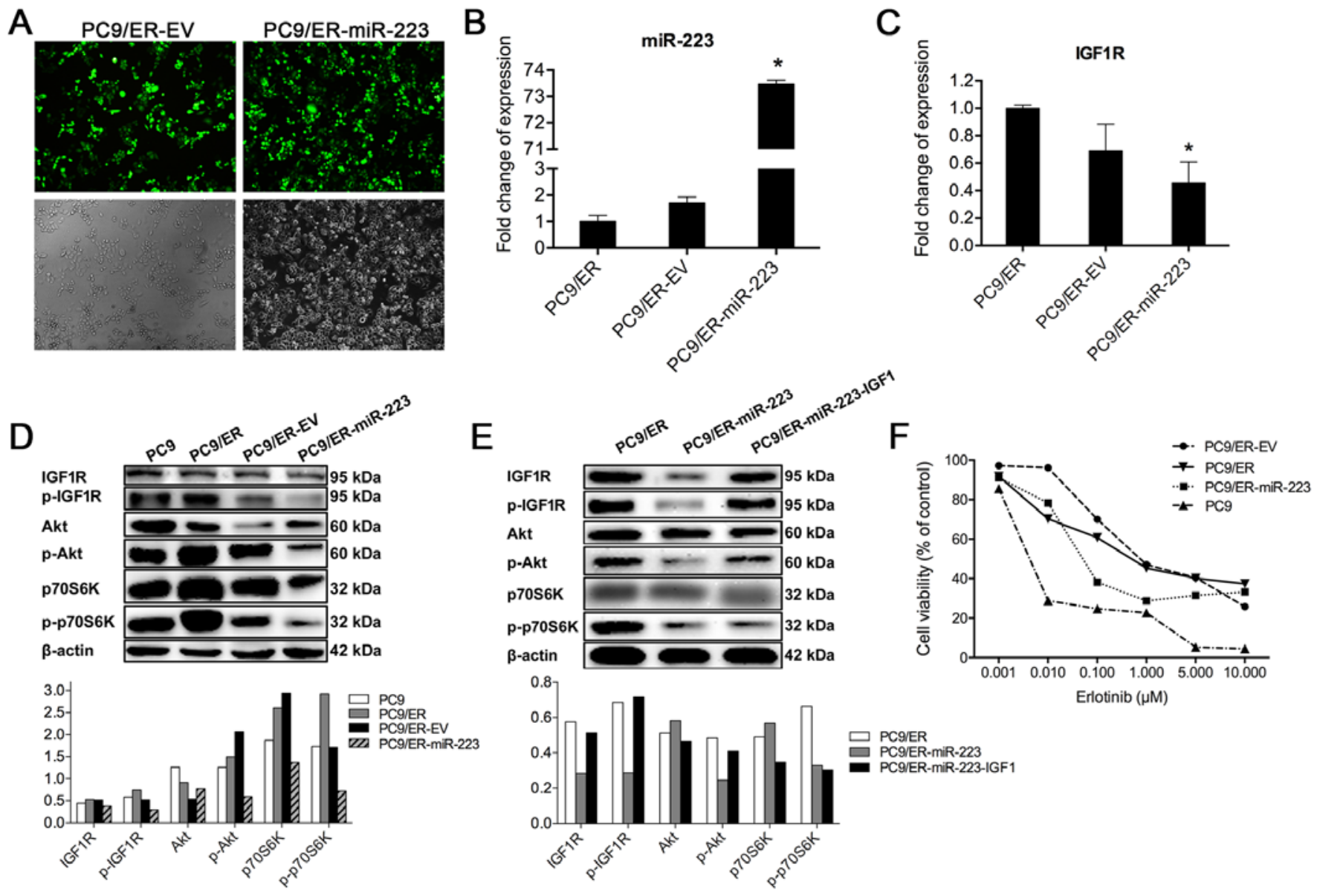

Figure 3. Inhibitory effect of miR-223 on IGF1R/PI3K/Akt signaling pathway in vitro. (A) The GV259-miR-223 lentiviral vector was packaged and delivered to PC9/ER cells. Transduction efficiency was evaluated based on the fluorescent GFP signal. Fluorescent GFP signal was stably observed $72 \mathrm{~h}$ after infection. EV for control vector-infected cells (right panel) and miR-223 for GV259-miR-223-infected cells (left panel) are shown. Magnification, x100. (B) miR-223 was overexpressed in PC9/ER cells as confirmed by real-time RT-PCR analysis. (C) Downregulation of IGF1R expression in PC9/ER-miR-223 cells was confirmed by real-time RT-PCR analysis. (D) IGF1R/Akt/p70S6K pathway was suppressed in the miR-223-overexpressing cells. Phosphorylation of Akt and p70S6K was significantly inhibited, according to western blot analysis. (E) miR-223 mediated inhibition of IGF1R/PI3K/Akt was reversed by agonist (IGF1) of IGF1R in miR-223 transfected cells. p-IGF1R and p-Atk were increased as shown in western blots. (F) The cytotoxicity and IC $_{50}$ values in PC9, PC9/ER, PC9/ER-EV, and PC9/ER-miR-223 cells. The cells were treated with the indicated concentrations of erlotinib for $72 \mathrm{~h}$ in medium containing $1 \% \mathrm{FBS}$. Cell viability and $\mathrm{IC}_{50}$ values were determined using the CCK8 assay.

might be caused by the binding of miR-223 to the 3'-UTR of IGF1R. Thus, IGF1R was identified as a direct target gene of miR-223 (Fig. 2B and C). To explore the relationship between miR-223 expression and erlotinib resistance, we also examined the expression level of miR-223 in both PC9/ER and HCC827/ER as well as in their parental cells. The analysis of data from real-time RT-PCR revealed that miR-223 level was decreased by $88 \%$ in PC9/ER cells, compared to that in PC9 cells (Fig. 2D). However, no significant difference in the expression levels of miR-223 was observed between HCC827 and HCC827/ER cells (Fig. 2E). Thus, we speculated that the downregulation of miR-223 expression may be involved in the acquired erlotinib resistance in PC9/ER cells.

Overexpression of miR-223 in PC9/ER cells. To evaluate the role of miR-223 in the development of acquired erlotinib resistance in PC9/ER cells, we introduced miR-223 mimic into this cell line. Because the lentiviral vector is a potent experimental tool for inducing the stable gain- and loss-offunction phenotypes caused by individual miRNAs, PC9/ER cells were infected with the lentiviral vector GV259 carrying hsa-miR-223. The infection efficiency, which can be estimated by GFP signals of the GV259 vector, was $~ 85 \%$ in the infected PC9/ER cells with either EV or miR-223 (Fig. 3A). While the levels of miR-223 were increased by 73.5 -fold in PC9/ER-miR-223 cells, compared to those in PC9/ER-EV cells, according to the results of real-time RT-PCR analysis (Fig. 3B). In our further experiments, PC9/ER-miR-223 was used as a stable cell line with overexpressed miR-223.

Inhibition of IGFIR/PI3K/Akt signaling pathway by overexpression of miR-223. To evaluate the effect of miR-223 on IGF1R expression, we examined the levels of IGF1R mRNA in PC9/ER-miR-223 cells via real-time RT-PCR. Results showed that IGF1R mRNA expression was reduced by $50 \%$ in PC9/ER-miR-223 cells, compared to PC9/ER cells (Fig. 3C). The overexpression of miR-223 also inhibited either the expression or the phosphorylation of IGF1R protein. It is well known that Akt is an essential protein kinase in the PI3K/Akt signaling pathway and it is also an 
important downstream molecule of IGF1R. Therefore, next, we determined the effect of miR-223 on Akt expression and its phosphorylation. We found that phosphorylation of Akt was reduced in PC9/ER-miR-223 cells. We further examined the expression of p70S6K, a key protein kinase in the mechanistic target of rapamycin (mTOR) signaling pathway, and its phosphorylation in PC9/ER-miR-223 cells. As shown in Fig. 3D, the phosphorylation levels of p70S6K was greatly reduced in PC9/ER-miR-223 cells, but significant changes in total p70S6K protein were not observed. Furthermore, our study also found that miR-223 mediated inhibition of the phosphorylation of either insulin-like growth factor 1 receptor (IGF1R) and its phosphorylation form (p-IGF1R) or Akt (p-Akt), except for 70S6K, which can be abolished by treatment with IGF1, an IGF1R agonist at dose of $10 \mathrm{ng} / \mathrm{ml}$ for $15 \mathrm{~min}$ (Fig. 3E). This result indicates that inactivation of IGF1R/PI3K/Akt/mTOR signaling pathway by miR-223 might be due to miR-223-mediated downregulation of IGF1R expression at both the mRNA and protein levels. Moreover, the cell viability of PC9, PC9/ER, PC9/ER-EV, and PC9/ ER-miR-223 was compared, according to the results of CCK8 assay. We found that after erlotinib treatment, the cell viability of PC9/ER-miR-223 was lower than that of PC9/ER and PC9/ER-EV cells, but higher than that of PC9 cells (Fig. 3F), indicating that the blockade of the IGF1R/PI3K/ Akt/mTOR signaling pathway is able to partially restore the sensitivity of the erlotinib-resistant cell lines. This partial reversion suggested that the activation of PI3K is not the sole mechanism for the resistance to EGFR-TKIs.

miR-223 partially reversed the acquired resistance to erlotinib by inhibiting the IGFIR/PI3K/Akt pathway in vivo. To verify the effect of miR-223 on the acquired resistance to erlotinib in vivo, a total of $5 \times 10^{6}$ PC9, PC9/ER, PC9/ER-EV or PC9/ER-miR-223 cells were subcutaneously injected into the front left legs of nude mice. The tumors appeared to be viable within one week after injection. When tumor volume reached $100 \mathrm{~mm}^{3}(14)$, nude mice bearing the xenograft tumors were treated with erlotinib $(30 \mathrm{mg} / \mathrm{kg} / \mathrm{d})(15)$ for two weeks. Then all mice were euthanized, except for those that had died early. Tumors were removed and weighed. Results showed that the average weight of tumors in the group with PC9/ER-miR-223 cells was $0.53 \pm 0.26 \mathrm{~g}$, which was significantly less than that of the mice injected with PC9/ER cells $(1.11 \pm 0.61 \mathrm{~g})$ (Fig. 4A and B). These results implied that the transfection of PC9/ER cells with miR-223 could partially restore the sensitivity of these cells to erlotinib. The histology of tumor xenografts was evaluated, using hematoxylin and eosin (H\&E) staining (Fig. 4A) and confocal laser-scanning microscopy. Both PC9/ER-miR-223 and PC9/ER-EV cells, which were visualized upon signals of GFP, can be clearly detected in frozen tumor sections via confocal laser scanning microscopy (Fig. 4C). The expression of miR-223 and IGF1R was determined via real-time RT-PCR. The expression levels of miR-223 in tumor xenografts from mice inoculated with PC9/ER-miR-223 cells were significantly higher than those from mice with PC9/ ER and PC9/ER-EV cells (Fig. 4D). The expression levels of IGF1R mRNA in tumor xenografts from mice with PC9/ ER-miR-223 were lower than those of mice with PC9/ER and PC9/ER-EV (Fig. 4E). These in vivo results were consistent with the results of in vitro experiments.

To investigate whether the inhibition of IGF1R can induce the inactivation of PI3K/Akt signaling pathway in vivo, we examined the activity of key molecules of IGF1R/PI3K/Akt signaling pathway in tumor tissues via western blot analysis. Our results showed that compared to the levels of p-IGF1R, p-Akt, and 70S6K in tumors derived from PC9/ER, the activity/phosphorylation of those molecules was inhibited in tumors derived from PC9/ER-miR-223 cells, but not in PC9/ER-EV derived tumors. The levels of p-IGF1R and p-Akt were decreased, and 70S6K was marginally reduced in the tumors derived from PC9 or PC9/ER cells after the treatment with erlotinib (Fig. 4F). Our results indicated that miR-223, rather than erlotinib, inhibits the activation of p70S6K, which is the downstream molecule of the PI3K/Akt pathway. Furthermore, miR-223 may partially reverse the resistance of PC9/ER cells to erlotinib by inhibiting the IGF1R/PI3K/Akt signaling pathway.

Detection of $\mathrm{CD} 133^{+}$population in cell lines of PC9ER and $P C$ 9. Since the CD133+ ${ }^{+}$subpopulation with cancer stem cell characteristics is associated with chemoresistance, we measured the percentage of $\mathrm{CD}_{133}{ }^{+}$cells in the cell lines of PC9/ER and PC9 by flow cytometry analysis. In PC9/ER, the percentage of $\mathrm{CD} 133^{+}$cells was $18.56 \%$ (Fig. $5 \mathrm{~A}-\mathrm{b}$ ) but was only $3.74 \%$ in the PC9 cell line (Fig. 5A-a). The ratio of $\mathrm{CD}_{133}{ }^{+}$cells (\%) between these two cell lines was 4.96 , with a statistically significant difference.

Inverse-induction with paclitaxel to enrich $\mathrm{CD}_{133^{+}}$subpopulation from PC9 cell line. In this study, we combined paclitaxel with a serum-free medium culture (inverse induction) to enrich $\mathrm{CD}_{133}{ }^{+}$subpopulation from PC9 cells. Briefly, PC9 cells were resuspended in a serum-free medium and grown into spheres (Fig. 5B-a). At the second passage, paclitaxel (100 nmol/l) was added to the culture. The medium was replaced with fresh medium $48 \mathrm{~h}$ later. After paclitaxel treatment, most of the treated cells died. Approximately two weeks after treatment, the surviving cells were able to gradually regrow into spheres (Fig. 5B-b). CD133 ${ }^{+}$cells in spheroids were then measured by flow cytometry. We found that the expression of CD133+ was $\sim 90 \%$ in the fourth generation of spheroids after inverse induction combined with paclitaxel treatment, suggesting that the surviving cell subpopulation after the inverse induction with paclitaxel treatment might highly express the CD133+ marker (Fig. 5C).

To further verify whether the enriched $\mathrm{CD} 133^{+}$cell subpopulation represents cancer stem cells (CSCs), the ability of tumor formation of these cells in vivo was examined. In vivo subcutaneous xenografts on nude mice showed that as few as a total of $5 \times 10^{4} \mathrm{PC} 9 / \mathrm{CD} 133^{+}$cells were able to generate a tumor the size of $\sim 2 \mathrm{~mm}$ in diameter, 5 days after inoculation, while an inoculation of $5 \times 10^{4}$ PC9 cells did not form a tumor, observed after 2 weeks. It took about one week to see a visible tumor after an inoculation of $5 \times 10^{6}$ PC9 cells into nude mice. The period of the time for observation in our study was 3 weeks, after which all mice were euthanized (Fig. 5D). Our animal data demonstrated that PC9/CD133+ cells have features of stemness with a stronger capability of tumorigenesis than PC9 cells. 

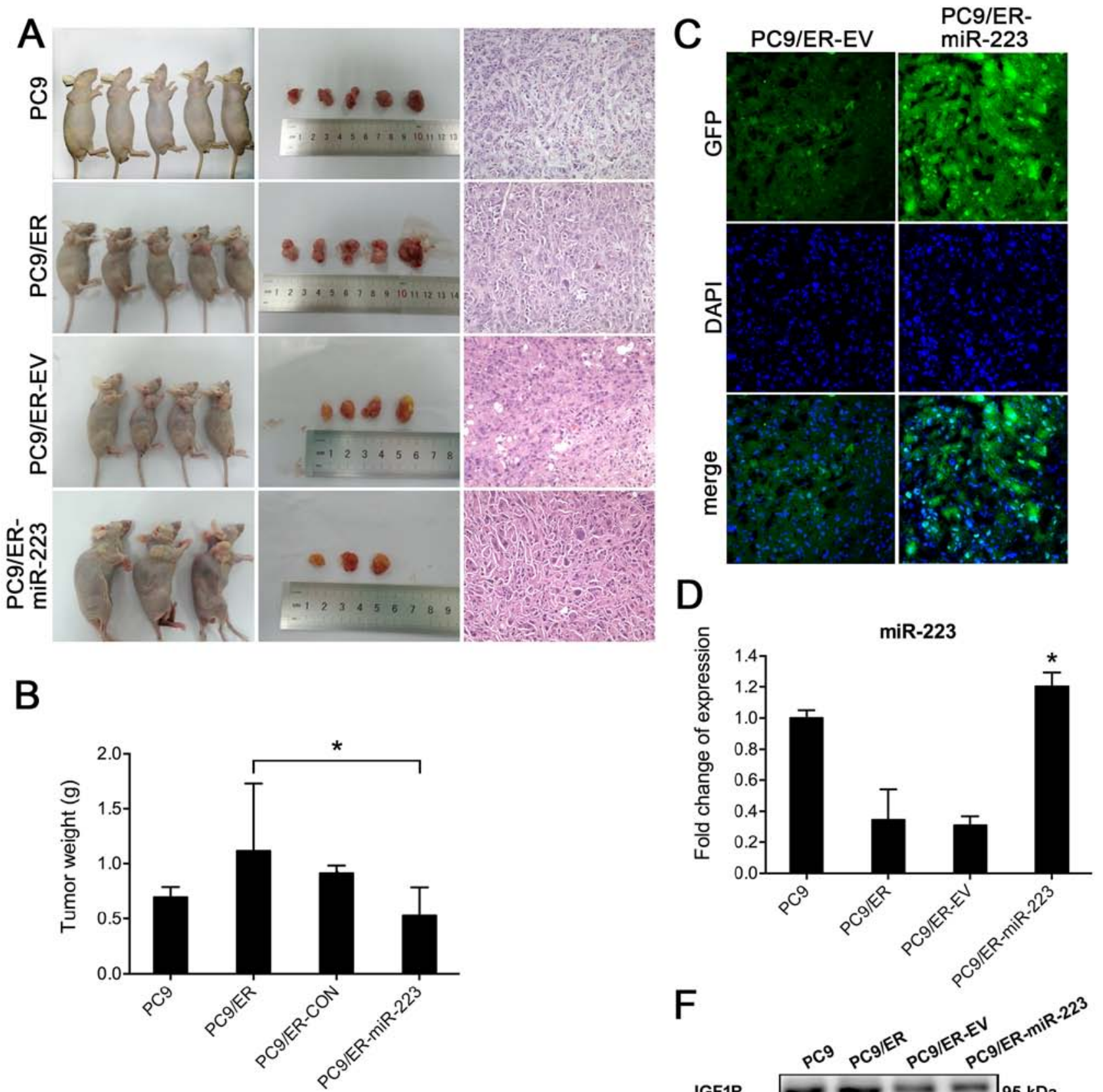

E
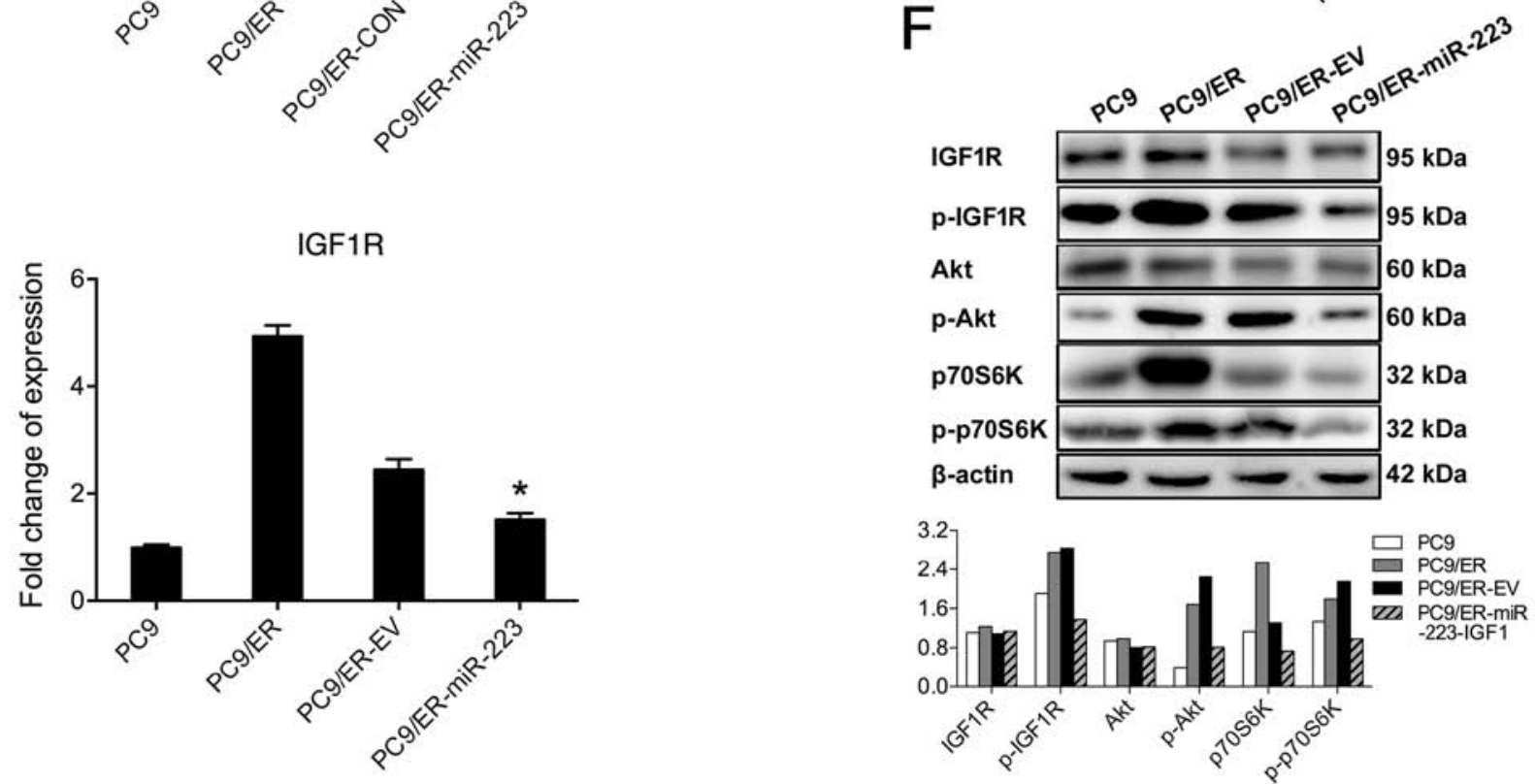

Figure 4. Inhibitory effect of miR-223 on IGF1R/PI3K/Akt signaling pathway in vivo. (A) The tumor sizes of nude mice in 4 groups (PC9, PC9/ER, PC9/ER-EV and PC9/ER-miR-223). H\&E staining of tumor xenografts from nude mice; magnification, $\mathrm{x} 100$. (B) Bar graphs show average tumor weight (g); ${ }^{*} \mathrm{P}<0.05$. (C) Detection of PC9/ER-miR-223 and PC9/ER-EV cells in frozen tumor sections using laser confocal microscopy, based on signals of green fluorescent protein. Nuclei were stained with 4,6-diamidino-2-phenylindole (DAPI); magnification, x100. (D and E) Expression levels of miR-223 and IGF1R were determined via real-time RT-PCR. (F) Western blot analysis of key molecules of IGF1R/PI3K/Akt/p70S6K signaling pathway in tumor tissues. 
A

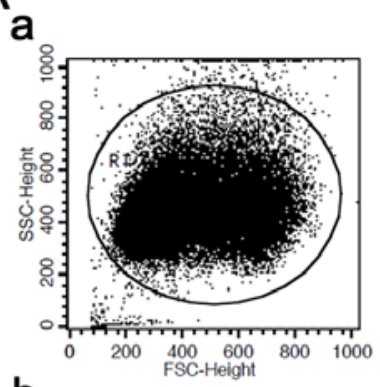

b

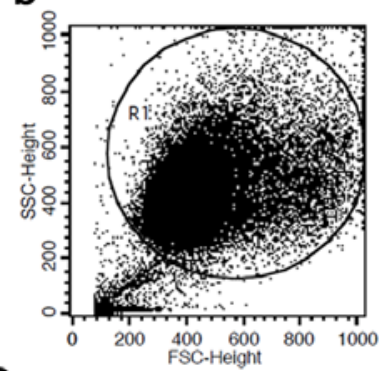

C

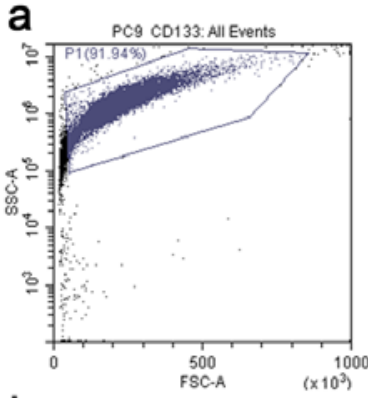

b

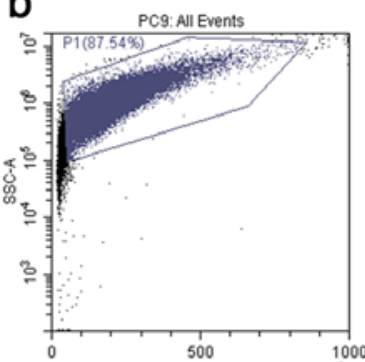

E

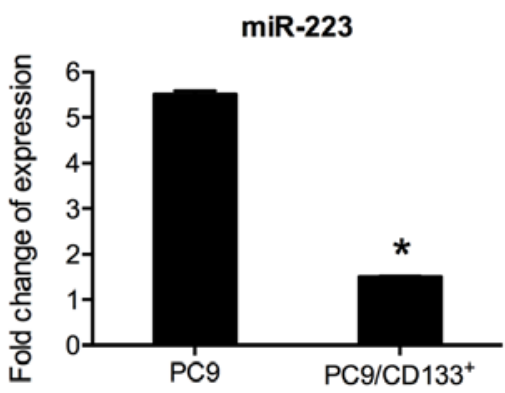

B
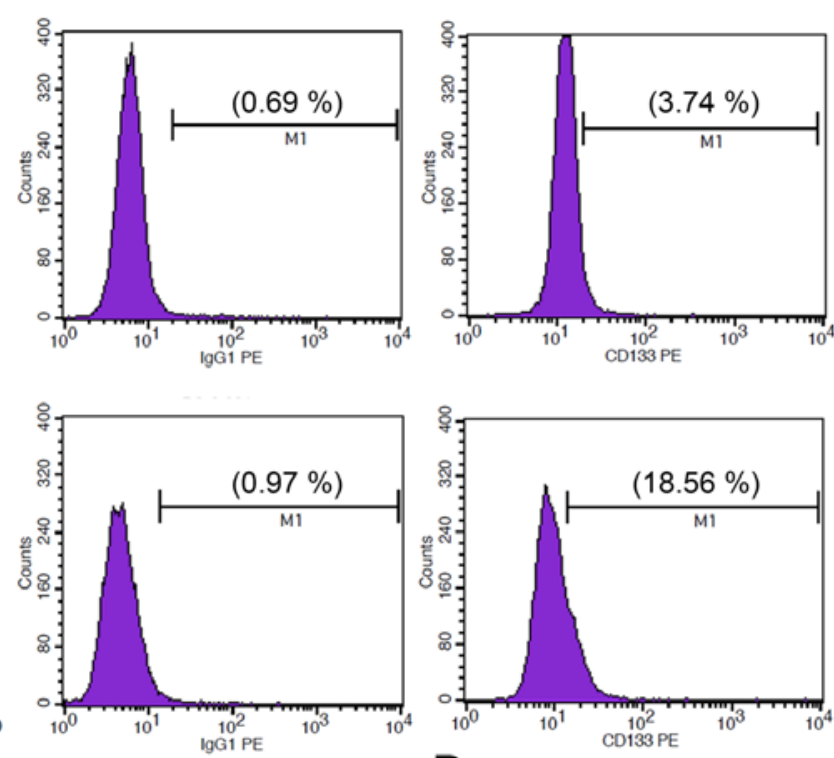

D

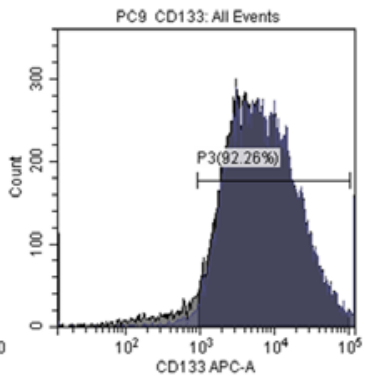

a

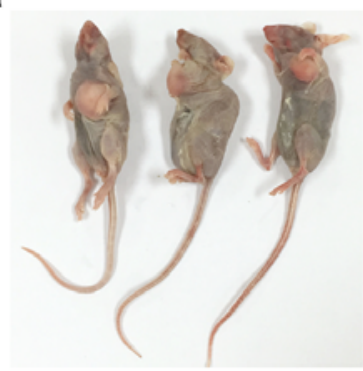

C
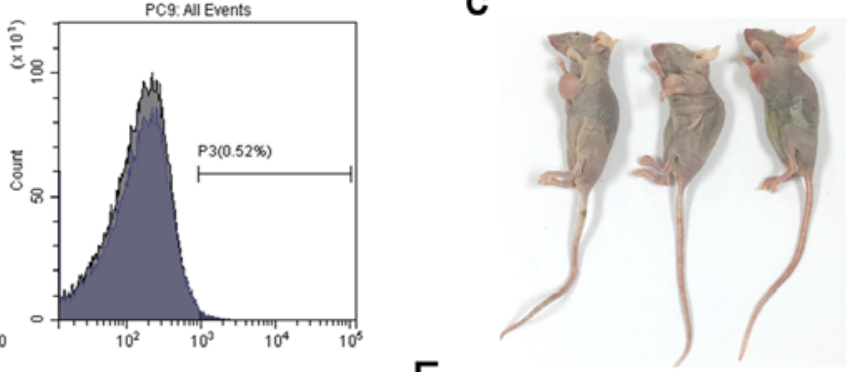

$\mathrm{F}$

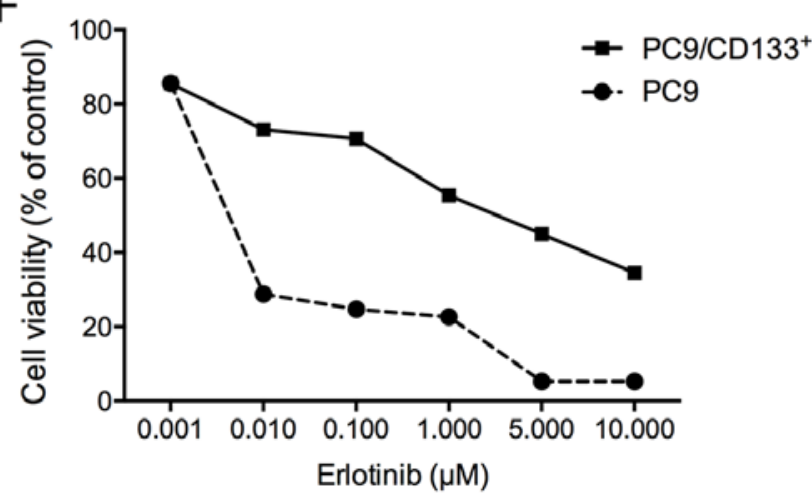

Figure 5. Isolation and expansion of $\mathrm{CD}_{133^{+}}$subpopulation for drug sensitivity testing. (A) Flow cytometry analysis of the percentage of $\mathrm{CD} 133^{+}$cells in PC9 (a) and PC9/ER (b) cell lines. (B-a) The morphology of the cells cultured in medium for stem cells, prior to paclitaxel treatment. Most were adherent cells; few were spheroids cells, with magnification, x100; (b) after paclitaxel treatment, most adherent cells died, and surviving cells regrew as spheroids, approximately two weeks after treatment, with magnification, x200. (C) Enrichment of CD133 ${ }^{+}$subpopulation by paclitaxel treatment, which was confirmed by flow cytometry analysis $\left(92.26 \% \mathrm{CD} 133^{+}\right.$cells in second generation of PC9 spheroids cells). (D) Ability of tumor formation in vivo between PC9/CD133 ${ }^{+}$

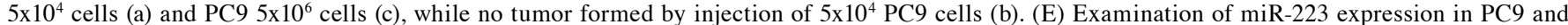
PC9/CD133 cells by real-time RT-PCR. Level of miR-223 was decreased by $72.6 \%$ in PC9/CD133 ${ }^{+}$cells, compared to that in PC9 cells. (F) Cytotoxicity and $\mathrm{IC}_{50}$ values of erlotinib in PC9 and PC9/CD133 cells. Cells were treated with indicated concentrations of erlotinib for $72 \mathrm{~h}$ in medium containing $1 \% \mathrm{FBS}$. Cell viability and $\mathrm{IC}_{50}$ values were determined using the CCK8 assay. 

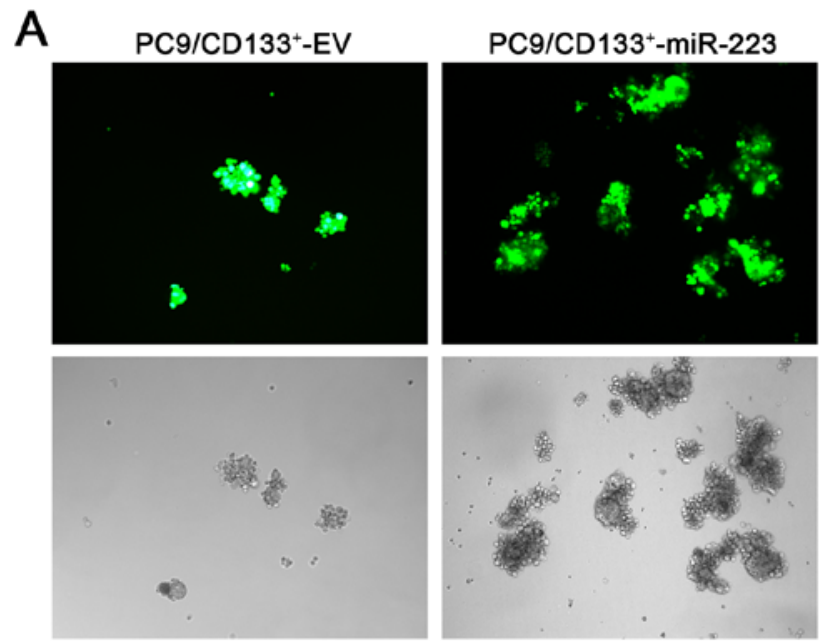

C

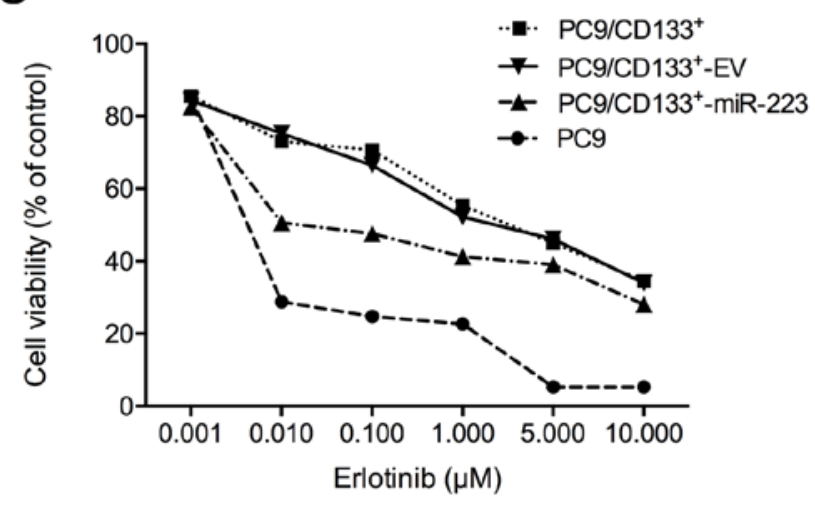

B

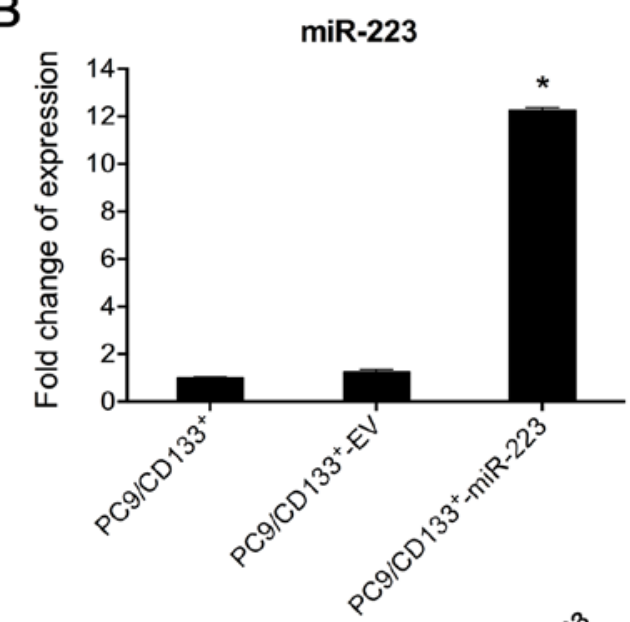

$\mathrm{D}$

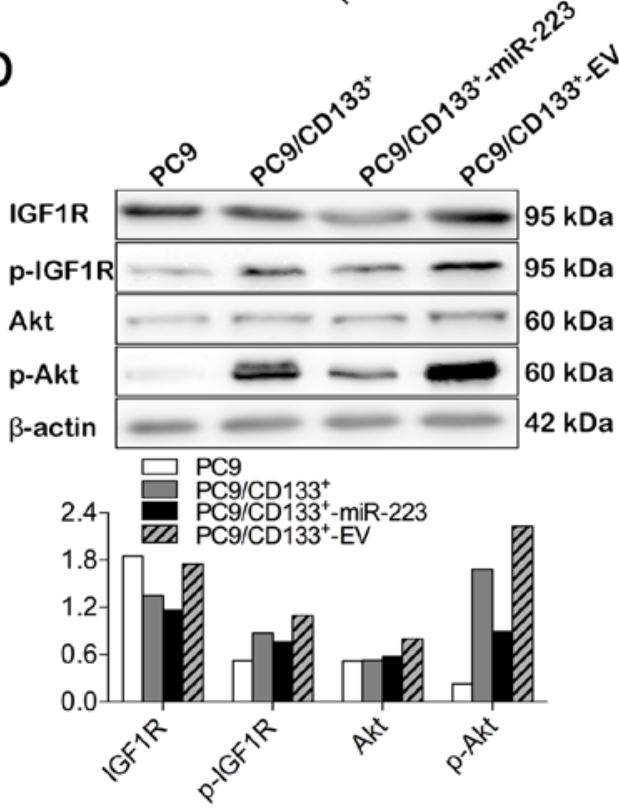

Figure 6. miR-223 inhibits the IGF1R/PI3K/Akt signaling pathway in CD133+ subpopulation cells. (A) The GV259-miR-223 lentiviral vector was packaged and delivered to PC9/CD133 ${ }^{+}$cells. Transduction efficiency was evaluated based on fluorescent GFP signal. Fluorescent GFP signal was stably observed $72 \mathrm{~h}$ after infection. EV for control vector-infected cells (a) and miR-223 for GV259-miR-223-infected cells (b). Magnification, x100. (B) Transduction efficiency was evaluated by real-time RT-PCR analysis, and expression of miR-223 in PC9/CD133+-miR-223 cells was 12.5 -fold that in PC9/CD133 ${ }^{+}$cells. (C) Cytotoxicity and $\mathrm{IC}_{50}$ values of erlotinib in PC9,PC9/CD133+, PC9/CD133+-miR-223, and PC9/CD133+-EV cells. Cells were treated with indicated concentrations of erlotinib for $72 \mathrm{~h}$ in medium containing $1 \% \mathrm{FBS}$. Cell viability and $\mathrm{IC}_{50}$ values in the treated cells were determined using the CCK8 assay. (D) Western blot analysis of the key molecules of IGF1R/PI3K/Akt signaling pathway in the erlotinib-treated $(0.01 \mu \mathrm{M}$ for $72 \mathrm{~h}) \mathrm{PC} 9, \mathrm{PC} 9 / \mathrm{CD} 133^{+}, \mathrm{PC} 9 / \mathrm{CD} 133^{+}$-miR-223, and PC9/CD133+-EV cells.

Determination of miR-223 expression in $\mathrm{PC} 9 / \mathrm{CD} 133^{+}$and PC9 cells as well as their erlotinib resistance. The expression level of miR-223 in PC9/CD133+ cells sorted by flow cytometry was determined using real-time RT-PCR. Results showed that miR-223 was downregulated by $72.6 \%$ in $\mathrm{PC} 9 / \mathrm{CD} 133^{+}$ cells, compared to that in PC9 cells (Fig. 5E). The dosedependent effect of erlotinib on cell viability was determined via the CCK8 assay. The $\mathrm{IC}_{50}$ value of erlotinib in $\mathrm{PC} 9 / \mathrm{CD} 133^{+}$ cells was 69.90-fold higher than that in PC9 cells (Fig. 5F), implying that $\mathrm{PC} 9 / \mathrm{CD} 133^{+}$cells are more resistant than $\mathrm{PC} 9$ cells to erlotinib.

Effects of miR-223 on IGFIR/PI3K/Akt signaling pathway in $\mathrm{PC} 9 / \mathrm{CD} 133^{+}$cells. To make a stably transfected cell line with miR-223, PC9/CD133 ${ }^{+}$cells, which were sorted by flow cytometry were grown in the culture medium of stem cells for 2 days. Then cells with good viability were selected for transfection of miR-223 or control EV (Fig. 6A). Transfection efficacy was confirmed using real-time RT-PCR. PCR data showed that miR-223 expression level in $\mathrm{PC} 9 / \mathrm{CD} 133^{+}$-miR-223 cells was 12.25 -fold higher than that in $\mathrm{PC} 9 / \mathrm{CD} 133^{+}$cells (Fig. 6B). $\mathrm{IC}_{50}$ values of erlotinib in both $\mathrm{PC} 9 / \mathrm{CD} 133^{+}-\mathrm{miR}-223$ and $\mathrm{PC} 9 / \mathrm{CD} 133^{+}$cells were calculated, based on data from CCK8 assay. Compared to PC $9 / \mathrm{CD} 133^{+}$cells, $\mathrm{IC}_{50}$ of erlotinib was decreased by $45 \%$ in $\mathrm{PC} 9 / \mathrm{CD} 133^{+}$-miR-223 cells (Fig. 6C), from which the resistance index was calculated as 0.46. As shown in Fig. 6D, the phosphorylation of IGF1R and Akt was inhibited in PC9/ CD133 ${ }^{+}$-miR-223 cells, compared to that in both $\mathrm{PC} 9 / \mathrm{CD} 133^{+}$ and $\mathrm{PC} 9 / \mathrm{CD} 133^{+}$-EV cells, implicating that upregulation of 
miR-223 expression in $\mathrm{PC} 9 / \mathrm{CD} 133^{+}$cells is able to inhibit the IGF1R/PI3K/Akt signaling pathway.

\section{Discussion}

Besides the mutation of the EGFR gene or amplification of the MET proto-oncogene, other mechanisms for the acquired resistance to erlotinib have recently been found. Our study showed that Akt, a key downstream molecule of the PI3K pathway, was constitutively active in PC9/ER and HCC827/ER cells. The important role of PI3K/Akt signaling pathway in the mechanisms underlying acquired resistance in lung cancer has been reported (18). In vitro experiments showed that the inhibitor of PI3K/Akt pathway can inhibit proliferation of cancer cells and induced apoptosis (19,20). PI3K inhibitors also markedly enhanced the sensitivity of NSCLC cells with high level of phosphorylated Akt to drug-induced apoptosis. As apoptosis deficiency is the principal mechanism of drug resistance in cancer cells, it has become a major goal to re-sensitize drug-resistant cells and kill them through overcoming defective apoptosis. PI3K-Akt signaling is known to promote survival under apoptotic stresses, so that the inhibition of PI3K/Akt signaling can block growth of cancer cells and promote their apoptosis (21-23). It has been reported that continuously activated PI3K signaling induces the resistance to EGFR-TKIs. Thus, the blockage of PI3K/Akt signaling overcame the resistance to EGFR-TKIs by inducing apoptosis of cancer cells via in vitro and in vivo models (24). Moreover, most cell models of acquired resistance exhibited continuous activity of PI3K signaling pathway (13,25-27). Our study also confirmed that PI3K/Akt signaling pathway is activated in either PC9/ER or HCC827/ER cells, indicating that PI3K/Akt signaling pathway may be associated with acquired erlotinib resistance in both cell lines.

Aberrant expression of miRNA is a common characteristic of human cancers, including NSCLC $(28,29)$. Recent studies have confirmed that miRNAs are involved in the modulation of the resistance of lung cancer cells to EGFR-TKIs. For example, miR-21 participates in the process of the acquired resistance to EGFR-TKIs in NSCLC, through downregulating PTEN and PDCD4 as well as activating PI3K/Akt pathway (30). The $\mathrm{miR}-134 / 487 \mathrm{~b} / 655$ cluster is involved in the regulation of drug resistance to gefitinib by targeting MAGI2 in lung adenocarcinoma cells (31). Another example showed that depletion of miR-205 induces erlotinib resistance (32). As for miR-223: in the literature, this miRNA has been confirmed to modulate multidrug resistance via the downregulation of ABCB1 in hepatocellular carcinoma (33). The suppression of miR-223 expression restored FBXW7 expression and the sensitivity of GC cells to trastuzumab (34). However, the role of miR-223 in resistance to EGFR-TKIs is rarely reported. IGF1R has been identified as a functional target of miR-223 and upregulation of miR-223 expression leads to inhibition of IGF1R expression $(10,35)$. In NSCLC, IGF1R acts as an important receptor tyrosine kinase and an upstream regulator of Akt (36), but no additional evidence supports the involvement of miR-223 in the acquired resistance of EGFR-TKIs in NSCLC. In this study, we assessed the expression levels of miR-223 in PC9/ ER and HCC827/ER cells and found that miR-223 expression was reduced by $88 \%$ in PC9/ER cells, compared to PC9 cells.
However, the expression of miR-223 was 1.5 times greater in HCC827/ER cells, compared to HCC827 cells. Moreover, the mRNA level of IGF1R measured by real-time RT-PCR was greatly increased in PC9/ER cells, compared to that in PC9 cells. Western blot analysis also demonstrated an increase in p-IGF1R induced by the treatment with $0.01 \mu \mathrm{M}$ erlotinib in PC9/ER cells, while the phosphorylation of IGF1R was inhibited in erlotinib-treated PC9 cells. In our study, overexpression of miR-223 in PC9/ER cells via lentiviral transduction inhibited the IGF1R/PI3K/Akt pathway and partially reversed the resistance of PC9/ER cells to erlotinib. Similar results were found in our in vivo study. Furthermore, miR-223-mediated inhibition of the phosphorylation of either IGF1R (p-IGF1R) or Akt (p-Akt), can be abolished by treatment with IGF1R agonist, suggesting that miR-223-mediated downregulation of IGF1R expression at mRNA and protein levels resulted in the inhibition of the IGF1R/PI3K/Akt/mTOR signaling pathway. These findings confirmed that the downregulation of miR-223 activates the IGF1R/PI3K/Akt pathway in PC9/ER cells and induces resistance to erlotinib.

Recently, CD133 has been identified as a biomarker of cancer stem cells in a variety of human tumors, including lung carcinoma (37-39). Eramo et al (4) confirmed that CD133+ subpopulation isolated from the tissue samples of lung carcinoma processed more cancer stem-cell-like characteristics, such as formation of tumor spheres in vitro and generation of tumors in nude mice, compared to CD133- cells. We found that the percentage of $\mathrm{CD} 133^{+}$subpopulation in PC9ER cells was 4.96 times that in PC9 cells. We used the method of inverse induction combined with paclitaxel to enrich the CD133+ subpopulation from the PC9 cell line. The $\mathrm{IC}_{50}$ values of erlotinib between $\mathrm{PC} 9$ and $\mathrm{PC} 9 / \mathrm{CD} 133^{+}$cells were compared. We found that $\mathrm{PC} / \mathrm{CD} 133^{+}$cells were more resistant to erlotinib, than PC9 cells. To clarify whether there is downregulation of miR-223 expression and activation of the IGF1R/PI3K/Akt signaling pathway in the $\mathrm{CD} 133^{+}$cell population, we examined miR-223 levels and the phosphorylation of key molecules of the IGF1R/PI3K/Akt pathway.

Our data revealed that downregulation of the miR-223 expression in $\mathrm{PC} / \mathrm{CD} 133^{+}$cells led to the activation of the IGF1R/PI3K/Akt signaling pathway, which may be the reason for the greater capacity of $\mathrm{CD}_{133^{+}}$cells for erlotinib resistance. It is still debated today whether $\mathrm{CD} 33^{+}$alone is the marker of CSCs. In our study, the $\mathrm{CD} 133^{+}$subpopulation, which was isolated from PC9 cells, displayed cancer stem celllike characteristics, including continuous expansion of tumor spheres (self-renewal) and pluripotent differentiation potential (differentiating into multiple cell types), a large capacity for drug resistance, and greater tumorigenic potential in vivo. Our findings of erlotinib resistance in the $\mathrm{CD}_{133^{+}}$subpopulation suggest that erlotinib treatment may not eradicate cancer stem cells in lung carcinoma, leading to a failure of molecular target therapy. Our study also revealed that the erlotinib resistance in $\mathrm{CD}_{133^{+}}$cells with cancer stem cell-like characteristics is associated with downregulation of miR-223 and activation of the IGF1R/PI3K/Akt signaling pathway. These results provide new insight into the mechanism underlying the resistance to EGFR-TKIs.

In this study, we developed the erlotinib-resistant cell lines HCC827/ER and PC9/ER by administering high-dose 
(1-5 $\mu \mathrm{M})$ pulses combined with continuous low-dose applications of erlotinib $(0.01 \mu \mathrm{M})$ for $>8$ months, which mimicked the mode of administration of erlotinib in the clinic. In most previous reports, cell models of NSCLC resistance to EGFRTKIs were established via stepwise escalation of EGFR-TKI concentrations. Acquired resistance associated with genetic changes, such as T790M EGFR mutation or MET amplification, was gained via the stepwise escalation method of EGFR-TKIs exposure after prolonged treatment $(13,40)$. In our study, neither T790M EGFR mutation nor MET amplification was observed in two erlotinib-resistant cell lines: PC9/ ER and HCC827/ER, in which PI3K/Akt signaling pathway was activated and an in-frame deletion in exon 19 of EGFR was detected. We also found that downregulation of miR-223 resulted in the activation of IGF1R/PI3K/Akt pathway in PC9/ER cells, leading to a secondary resistance of these cells to erlotinib. In contrast, the expression of miR-223 was 1.5 times greater in HCC827/ER cells than in HCC827 cells. To explain these contradictory findings, we examined PTEN expression in HCC827/ER and PC9/ER cells. Results showed that PTEN mRNA was decreased in HCC827/ER cells, but slightly increased in PC9/ER cells, compared to the corresponding parental cells. Similar results for the PTEN protein were also obtained. Therefore, the resistance of HCC827/ER cells to erlotinib may be considered a result of the loss of PTEN, thereby activating the PI3K/Akt pathway. Our findings revealed different mechanisms of resistance to EGFR-TKIs in lung adenocarcinoma, harboring mutated oncogene or loss of tumor suppressor gene, although the establishment of these resistant cell lines used the same culture conditions and methods. The mechanisms underlying the secondary resistance to EGFR-TKIs in patients with lung cancer may be more complex, because of the complexity of the tumor microenvironment and differences between individuals. As a result, for precise individualized therapy, it is necessary to explore additional mechanisms for resistance to EGFR-TKIs.

In conclusion, miR-223 expression was downregulated in PC9/ER cells and $\mathrm{PC} 9 / \mathrm{CD} 133^{+}$cells, leading to the activation of the IGF1R/PI3K/Akt signaling pathway in these cells, which may be one mechanism responsible for the resistance of PC9/ER cells and PC9/CD133 ${ }^{+}$cells to erlotinib.

\section{Acknowledgements}

This study was supported in part by grants from the National Natural Science Foundation of China (81172070 and 81071786) and the National High Technology Research and Development Program of China (2008AA02Z104).

\section{References}

1. Jemal A, Siegel R, Ward E, Hao Y, Xu J, Murray T and Thun MJ: Cancer statistics, 2008. CA Cancer J Clin 58: 71-96, 2008.

2. Bezjak A, Tu D, Seymour L, Clark G, Trajkovic A, Zukin M, Ayoub J, Lago S, de Albuquerque Ribeiro R, Gerogianni A, et al; National Cancer Institute of Canada Clinical Trials Group Study BR.21: Symptom improvement in lung cancer patients treated with erlotinib: Quality of life analysis of the National Cancer Institute of Canada Clinical Trials Group Study BR.21. J Clin Oncol 24: 3831-3837, 2006.

3. Nguyen KS, Kobayashi S and Costa DB: Acquired resistance to epidermal growth factor receptor tyrosine kinase inhibitors in non-small-cell lung cancers dependent on the epidermal growth factor receptor pathway. Clin Lung Cancer 10: 281-289, 2009.
4. Eramo A, Lotti F, Sette G, Pilozzi E, Biffoni M, Di Virgilio A, Conticello C, Ruco L, Peschle C and De Maria R: Identification and expansion of the tumorigenic lung cancer stem cell population. Cell Death Differ 15: 504-514, 2008

5. Levina V, Marrangoni A, Wang T, Parikh S, Su Y, Herberman R, Lokshin A and Gorelik E: Elimination of human lung cancer stem cells through targeting of the stem cell factor-c-kit autocrine signaling loop. Cancer Res 70: 338-346, 2010.

6. Sun FF, Hu YH, Xiong LP, Tu XY, Zhao JH, Chen SS, Song J and Ye XQ: Enhanced expression of stem cell markers and drug resistance in sphere-forming non-small cell lung cancer cells. Int J Clin Exp Pathol 8: 6287-6300, 2015.

7. Hassan SS, Romero R, Pineles B, Tarca AL, Montenegro D, Erez O, Mittal P, Kusanovic JP, Mazaki-Tovi S, Espinoza J, et al: MicroRNA expression profiling of the human uterine cervix after term labor and delivery. Am J Obstet Gynecol 202: 80.e1-80.e8, 2010.

8. Geng Q, Fan T, Zhang B, Wang W, Xu Y and Hu H: Five microRNAs in plasma as novel biomarkers for screening of early-stage non-small cell lung cancer. Respir Res 15: 149, 2014.

9. Wang H, Wang L, Wu Z, Sun R, Jin H, Ma J, Liu L, Ling R, Yi J, Wang L, et al: Three dysregulated microRNAs in serum as novel biomarkers for gastric cancer screening. Med Oncol 31: 298, 2014.

10. Nian W, Ao X, Wu Y, Huang Y, Shao J, Wang Y, Chen Z, Chen F and Wang D: miR-223 functions as a potent tumor suppressor of the Lewis lung carcinoma cell line by targeting insulin-like growth factor-1 receptor and cyclin-dependent kinase 2 . Oncol Lett 6: 359-366, 2013.

11. Miller TE, Ghoshal K, Ramaswamy B, Roy S, Datta J, Shapiro CL, Jacob S and Majumder S: MicroRNA-221/222 confers tamoxifen resistance in breast cancer by targeting p27Kip1. J Biol Chem 283: 29897-29903, 2008.

12. Shen H, Zhu F, Liu J, Xu T, Pei D, Wang R, Qian Y, Li Q, Wang L, Shi Z, et al: Alteration in Mir-21/PTEN expression modulates gefitinib resistance in non-small cell lung cancer. PLoS One 9: e103305, 2014.

13. Engelman JA, Zejnullahu K, Mitsudomi T, Song Y, Hyland C, Park JO, Lindeman N, Gale CM, Zhao X, Christensen J, et al: MET amplification leads to gefitinib resistance in lung cancer by activating ERBB3 signaling. Science 316: 1039-1043, 2007.

14. Ma H, Yao Q, Zhang AM, Lin S, Wang XX, Wu L, Sun JG and Chen ZT: The effects of artesunate on the expression of EGFR and ABCG2 in A549 human lung cancer cells and a xenograft model. Molecules 16: 10556-10569, 2011.

15. Mu XY, Dong XL, Sun J, Ni YH, Dong Z, Li XL, Sun EL, Yi Z and Li G: Simultaneous blockage of epidermal growth factor receptor and cyclooxygenase-2 in a human xenotransplanted lung cancer model. Asian Pac J Cancer Prev 15: 69-73, 2014.

16. Guix M, Faber AC, Wang SE, Olivares MG, Song Y, Qu S, Rinehart C, Seidel B, Yee D, Arteaga CL, et al: Acquired resistance to EGFR tyrosine kinase inhibitors in cancer cells is mediated by loss of IGF-binding proteins. J Clin Invest 118: 2609-2619, 2008.

17. Choi YJ, Park GM, Rho JK, Kim SY, So GS, Kim HR, Choi CM and Lee JC: Role of IGF-binding protein 3 in the resistance of EGFR mutant lung cancer cells to EGFR-tyrosine kinase inhibitors. PLoS One 8: e81393, 2013.

18. Liu LZ, Zhou XD, Qian G, Shi X, Fang J and Jiang BH: AKT1 amplification regulates cisplatin resistance in human lung cancer cells through the mammalian target of rapamycin/p70S6K1 pathway. Cancer Res 67: 6325-6332, 2007.

19. Alexia C, Bras M, Fallot G, Vadrot N, Daniel F, Lasfer M, Tamouza $\mathrm{H}$ and Groyer A: Pleiotropic effects of PI-3' kinase/Akt signaling in human hepatoma cell proliferation and drug-induced apoptosis. Ann NY Acad Sci 1090: 1-17, 2006.

20. Poh TW and Pervaiz S: LY294002 and LY303511 sensitize tumor cells to drug-induced apoptosis via intracellular hydrogen peroxide production independent of the phosphoinositide 3-kinase-Akt pathway. Cancer Res 65: 6264-6274, 2005.

21. Brozovic A and Osmak M: Activation of mitogen-activated protein kinases by cisplatin and their role in cisplatin-resistance. Cancer Lett 251: 1-16, 2007.

22. Corradetti MN and Guan KL: Upstream of the mammalian target of rapamycin: Do all roads pass through mTOR? Oncogene 25: 6347-6360, 2006.

23. Lekmine F, Uddin S, Sassano A, Parmar S, Brachmann SM, Majchrzak B, Sonenberg N, Hay N, Fish EN and Platanias LC: Activation of the p70 S6 kinase and phosphorylation of the 4E-BP1 repressor of mRNA translation by type I interferons. J Biol Chem 278: 27772-27780, 2003. 
24. Donev IS, Wang W, Yamada T, Li Q, Takeuchi S, Matsumoto K, Yamori T, Nishioka Y, Sone S and Yano S: Transient PI3K inhibition induces apoptosis and overcomes HGF-mediated resistance to EGFR-TKIs in EGFR mutant lung cancer. Clin Cancer Res 17: 2260-2269, 2011.

25. Ogino A, Kitao H, Hirano S, Uchida A, Ishiai M, Kozuki T, Takigawa N, Takata M, Kiura K and Tanimoto M: Emergence of epidermal growth factor receptor T790M mutation during chronic exposure to gefitinib in a non small cell lung cancer cell line. Cancer Res 67: 7807-7814, 2007.

26. Engelman JA, Mukohara T, Zejnullahu K, Lifshits E, Borrás AM, Gale CM, Naumov GN, Yeap BY, Jarrell E, Sun J, et al: Allelic dilution obscures detection of a biologically significant resistance mutation in EGFR-amplified lung cancer. J Clin Invest 116: 2695-2706, 2006.

27. Li H, Schmid-Bindert G, Wang D, Zhao Y, Yang X, Su B and Zhou C: Blocking the PI3K/AKT and MEK/ERK signaling pathways can overcome gefitinib-resistance in non-small cell lung cancer cell lines. Adv Med Sci 56: 275-284, 2011.

28. Du L and Pertsemlidis A: microRNAs and lung cancer: Tumors and 22-mers. Cancer Metastasis Rev 29: 109-122, 2010.

29. Gibson NW: Engineered microRNA therapeutics. J R Coll Physicians Edinb 44: 196-200, 2014.

30. Li B, Ren S, Li X, Wang Y, Garfield D, Zhou S, Chen X, Su C, Chen M, Kuang P, et al: MiR-21 overexpression is associated with acquired resistance of EGFR-TKI in non-small cell lung cancer. Lung Cancer 83: 146-153, 2014.

31. Kitamura K, Seike M, Okano T, Matsuda K, Miyanaga A, Mizutani H, Noro R, Minegishi Y, Kubota K and Gemma A: MiR-134/487b/655 cluster regulates TGF- $\beta$-induced epithelialmesenchymal transition and drug resistance to gefitinib by targeting MAGI2 in lung adenocarcinoma cells. Mol Cancer Ther 13: 444-453, 2014.

32. Park KS, Raffeld M, Moon YW, Xi L, Bianco C, Pham T, Lee LC, Mitsudomi T, Yatabe Y, Okamoto I, et al: CRIPTO1 expression in EGFR-mutant NSCLC elicits intrinsic EGFR-inhibitor resistance. J Clin Invest 124: 3003-3015, 2014.
33. Yang T, Zheng ZM, Li XN, Li ZF, Wang Y, Geng YF, Bai L and Zhang XB: MiR-223 modulates multidrug resistance via downregulation of $\mathrm{ABCB} 1$ in hepatocellular carcinoma cells. Exp Biol Med (Maywood) 238: 1024-1032, 2013.

34. Eto K, Iwatsuki M, Watanabe M, Ishimoto T, Ida S, Imamura Y, Iwagami S, Baba Y, Sakamoto Y, Miyamoto Y, et al: The sensitivity of gastric cancer to trastuzumab is regulated by the miR-223/FBXW7 pathway. Int J Cancer 136: 1537-1545, 2015.

35. Jia CY, Li HH, Zhu XC, Dong YW, Fu D, Zhao QL, Wu W and Wu XZ: MiR-223 suppresses cell proliferation by targeting IGF-1R. PLoS One 6: e27008, 2011.

36. Allen GW, Saba C, Armstrong EA, Huang SM, Benavente S, Ludwig DL, Hicklin DJ and Harari PM: Insulin-like growth factor-I receptor signaling blockade combined with radiation. Cancer Res 67: 1155-1162, 2007.

37. Atashpour S, Fouladdel S, Movahhed TK, Barzegar E, Ghahremani MH, Ostad SN and Azizi E: Quercetin induces cell cycle arrest and apoptosis in CD133(+) cancer stem cells of human colorectal HT29 cancer cell line and enhances anticancer effects of doxorubicin. Iran J Basic Med Sci 18: 635-643, 2015.

38. Miki J, Furusato B, Li H, Gu Y, Takahashi H, Egawa S, Sesterhenn IA, McLeod DG, Srivastava S and Rhim JS: Identification of putative stem cell markers, CD133 and CXCR4, in hTERT-immortalized primary nonmalignant and malignant tumor-derived human prostate epithelial cell lines and in prostate cancer specimens. Cancer Res 67: 3153-3161, 2007.

39. Kim CK, Kim SK, Yang YH, Lee MS, Yoon JH and Park CI: A case of recurrent infantile polycystic kidney associated with hydrops fetalis. Yonsei Med J 30: 95-103, 1989.

40. Suda K, Murakami I, Katayama T, Tomizawa K, Osada H, Sekido Y, Maehara Y, Yatabe Y and Mitsudomi T: Reciprocal and complementary role of MET amplification and EGFR T790M mutation in acquired resistance to kinase inhibitors in lung cancer. Clin Cancer Res 16: 5489-5498, 2010. 